\title{
Solutions of Free Higher Spins in AdS
}

\author{
H. Lü ${ }^{1,2}$ and Kai-Nan Shao ${ }^{3}$ \\ ${ }^{1}$ China Economics and Management Academy \\ Central University of Finance and Economics, Beijing 100081 \\ ${ }^{2}$ Institute for Advanced Study, Shenzhen University \\ Nanhai Ave 3688, Shenzhen 518060 \\ ${ }^{3}$ Zheijiang Institute of Modern Physics \\ Department of Physics, Zheijiang University, Hangzhou 310027
}

\begin{abstract}
We consider free massive and massless higher integer spins in AdS backgrounds in general $D$ dimensions. We obtain the solutions corresponding to the highest-weight state of the spin- $\ell$ representations of the $S O(2, D-1)$ isometry groups. The solution for the spin- $\ell$ field is expressed recursively in terms of that for the spin- $(\ell-1)$. Thus starting from the explicit spin-0, all the higher-spin solutions can be obtained. These solutions allow us to derive the generalized Breitenlohner-Freedman bound, and analyze the asymptotic falloffs. In particular, solutions with negative mass square in general have falloffs slower than those of the Schwarzschild AdS black holes in the AdS boundaries.
\end{abstract}


There has been continuing interest in higher spins since the early days of quantum field theory. The non-interacting integer higher-spin fields can be defined by the Fierz-Pauli conditions [1], namely the Klein-Gordan equation

$$
\left(\square-\left(M^{(\ell)}\right)^{2}\right) \phi_{\mu_{1} \cdots \mu_{\ell}}^{(\ell)}=0
$$

together with the transverse and traceless conditions

$$
\nabla^{\mu_{1}} \phi_{\mu_{1} \cdots \mu_{\ell}}^{(\ell)}=0, \quad g^{\mu_{1} \mu_{2}} \phi_{\mu_{1} \cdots \mu_{\ell}}^{(\ell)}=0
$$

where $\phi_{\mu_{1} \cdots \mu_{\ell}}^{(\ell)}$ are totally symmetric. The Lagrangian formulations for the free massive and massless higher-spin fields were obtained in [2] and [3] respectively.

It is natural to consider higher-spin gravity theory, which turns out to be inconsistent in Einstein gravity, which admits the Minkowski vacuum [4]. However, when a negative cosmological constant is introduced with an anti-de Sitter (AdS) vacuum, the interacting theory of gravity and higher spins can be consistently defined, at least at the level of equations of motion [5]. Considerable interest has since been paid to the subject. The importance of higher-spin gravities is apparent in the context of string theory in the tensionless limit where the infinite tower of higher-spin string excitations become massless. From the point of view of the AdS/CFT correspondence, gravities coupled to higher spins are expected to be the gravitational dual of the weakly-coupled conformal field theories. Here we give a few references [6]-[15] and reviews [16]-[20], which are by no means all inclusive.

When the theory has a cosmological constant, the definition of the mass in the KleinGordan equation is shifted by a constant $\left(M_{\text {AdS }}^{(\ell)}\right)^{2}$ that depends on the dimension $D$, spin $\ell$ and the cosmological constant. The Klein-Gordan equation becomes

$$
\left(\square-\left(M_{\mathrm{AdS}}^{(\ell)}\right)^{2}-\left(M^{(\ell)}\right)^{2}\right) \phi_{\mu_{1} \cdots \mu_{\ell}}^{(\ell)}=0,
$$

with the same transverse and traceless conditions (2). In the massless limit $M^{(\ell)}=0$, there are additional gauge symmetries. The gauge invariant equation of motion is given by [21]

$$
\begin{aligned}
& \square \phi_{\mu_{1} \cdots \mu_{s}}^{(\ell)}-\ell \nabla_{\left(\mu_{1}\right.}\left(\nabla \cdot \phi^{(\ell)}\right)_{\left.\mu_{2} \cdots \mu_{\ell}\right)}+\frac{1}{2} \ell(\ell-1) \nabla_{\left(\mu_{1}\right.} \nabla_{\mu_{2}} \phi_{\left.\mu_{3} \cdots \mu_{\ell}\right) \lambda}^{(\ell)}{ }^{\lambda} \\
& -\left(M_{\mathrm{AdS}}^{(\ell)}\right)^{2} \phi_{\mu_{1} \cdots \mu_{\ell}}^{(\ell)}-\left(\widetilde{M}_{\mathrm{AdS}}^{(\ell)}\right)^{2} g_{\left(\mu_{1} \mu_{2}\right.} \phi_{\left.\mu_{3} \cdots \mu_{\ell} \lambda\right)}^{(\ell)}=0,
\end{aligned}
$$

where

$$
\left(M_{\mathrm{AdS}}^{(\ell)}\right)^{2}=\frac{(\ell-2)(D-1)+(\ell-1)(\ell-4)}{L^{2}}, \quad\left(\widetilde{M}_{\mathrm{AdS}}^{(\ell)}\right)^{2}=\frac{\ell(\ell-1)}{L^{2}} .
$$

Here $L$ denotes the the "radius" of the AdS space-time. The gauge transformation rule is given by

$$
\delta \phi_{\mu_{1} \cdots \mu_{\ell}}^{(\ell)}=\ell \nabla_{\left(\mu_{1}\right.} \epsilon_{\left.\mu_{2} \cdots \mu_{\ell}\right)},
$$


where $\epsilon$ is totally symmetric and traceless. For $\ell=0$, the transverse and traceless conditions do not apply; for $\ell=1$, only the transverse condition is relevant. Interestingly, as we show in the appendix, for $\ell=2$ and 3 , we can impose some appropriate gauge fixing such that the transverse and traceless conditions come out naturally. However, for $\ell \geq 4$, the conditions (2) arise in a more subtle manner.

In this paper we are constructing explicit solutions of (3) with (2) imposed in arbitrary dimensions for arbitrary spin $\ell$. The $\ell \geq 1$ solutions form finite representations under the $S O(2, D-1)$ isometry group of $\mathrm{AdS}_{D}$. To obtain the full spin- $\ell$ representation, we can start with the solution associated with the highest-weight state, which is defined to be the eigenstate of the Cartan generators, annihilated by all the positive-root generators. Once such a solution is constructed, the full representation can be generated by acting repetitively on it with all possible negative-root generators. This method was first used to construct explicit graviton modes in three-dimensional topologically massive gravity [22]. Massless and massive graviton modes in higher-curvature extended gravities in four [23] and general dimensions [24] were also constructed. The aim of this paper is to employ this technique to obtain explicit solutions for free higher spins in general dimensions.

To construct explicit solutions, it is necessary to select appropriate coordinates. We shall write the the $\mathrm{AdS}_{D}$ metric in global coordinates. The metric for the foliating sphere is written in such a way that the $U(1)$ coordinates of the corresponding Cartan generators of the isometry group are manifest. Thus we choose the following AdS metric:

$$
d s^{2}=L^{2}\left(-\cosh ^{2} \rho d \tau^{2}+d \rho^{2}+\sinh ^{2} \rho d \Omega_{D-2}^{2}\right) .
$$

For odd $D=2 n+1$ dimensions, the foliating $S^{D-2}$ is given by

$$
\begin{aligned}
d \Omega_{D-2}^{2}= & d \theta_{1}^{2}+\cos \theta_{1} d \phi_{n}^{2}+\sin ^{2} \theta_{1}\left(d \theta_{2}^{2}+\cos ^{2} \theta_{2} d \phi_{n-1}^{2}\right. \\
& \left.+\sin ^{2} \theta_{2}\left(\cdots\left(d \theta_{n-1}^{2}+\cos \theta_{n-1} d \phi_{2}^{2}+\sin \theta_{n-1} d \phi_{1}^{2}\right)\right)\right) .
\end{aligned}
$$

For even $D=2 n$ dimensions, the metric takes the same form but with $\phi_{n}=0$. The full set of the $S O(2, D-1)$ Killing vectors for these AdS metrics were discussed in [24]. In particular, the Cartan generators can be conveniently chosen as

$$
H_{0}=\mathrm{i} \frac{\partial}{\partial \tau}, \quad H_{i}=\mathrm{i} \frac{\partial}{\partial \phi_{i}}, \quad i=1,2, \cdots,\left[\frac{D-1}{2}\right] .
$$

Consequently, the positive and negative root generators $\left(E_{\vec{\alpha}_{x}}, E_{-\vec{\alpha}_{x}}\right)$ can all be specified uniquely. These generators satisfy the $s o(2, D-1)$ algebra, namely

$$
\left[H_{i}, H_{j}\right]=0, \quad\left[H_{i}, E_{\vec{a}_{x}}\right]=\vec{\alpha}_{x}^{i} E_{\vec{\alpha}_{x}},
$$




$$
\left[E_{-\vec{\alpha}_{x}}, E_{\vec{\alpha}_{x}}\right]=\frac{2}{\left|\vec{\alpha}_{x}\right|^{2}} \vec{\alpha}_{x}^{i} H_{i}, \quad i=0,1,2, \cdots\left[\frac{D-1}{2}\right] .
$$

The procedure to obtain the explicit form of these generators in the above coordinates were given in [24].

We are looking for the spin- $\ell$ solution $\psi_{\mu_{1} \cdots \mu_{\ell}}^{(\ell)}$ that is the highest-weight state under the $S O(2, D-1)$ isometry group. It is defined by

$$
\begin{aligned}
& \left(H_{0}-E_{0}^{(\ell)}\right) \psi_{\mu_{1} \cdots \mu_{\ell}}^{(\ell)}=0, \quad\left(H_{i}-h_{i}\right) \psi_{\mu_{1} \cdots \mu_{\ell}}^{(\ell)}=0 \quad \text { for } \quad i=1,2, \cdots, \\
& E_{\vec{\alpha}_{x}} \psi_{\mu_{1} \cdots \mu_{\ell}}^{(\ell)}=0, \quad \text { for all simple roots } \vec{\alpha}_{x} \text { and hence all positive roots. }
\end{aligned}
$$

We use $\psi$ to denote the naturally-complex solutions, whilst $\phi$ is the real or imaginary part of $\psi$. The action of the generators on the solution $\psi_{\mu_{1} \cdots \mu_{\ell}}^{(\ell)}$ is as Lie derivative. The field $\psi_{\mu_{1} \cdots \mu_{\ell}}^{(\ell)}$ is totally symmetric, satisfying the transverse and traceless condition, namely

$$
g^{\mu_{1} \mu_{2}} \psi_{\mu_{1} \mu_{2} \cdots \mu_{\ell}}^{(\ell)}=0, \quad \nabla^{\mu_{1}} \psi_{\mu_{1} \mu_{2} \cdots \mu_{\ell}}^{(\ell)}=0 .
$$

Note that the Casimir operator $\mathcal{E}$ is related to the covariant Laplace operator $\Delta$ as follows

$$
\mathcal{E}=\sum_{i} H_{i} H_{i}+\sum_{x} \frac{1}{2}\left|\alpha_{x}\right|^{2}\left(E_{\alpha_{x}} E_{-\alpha_{x}}+E_{-\alpha_{x}} E_{\alpha_{x}}\right)=-L^{2} \Delta,
$$

where the two sums are over all the Cartan and root generators respectively. The Laplace acting on the spin- $\ell$ field is given by

$$
\begin{aligned}
\Delta \psi_{\mu_{1} \cdots \mu_{\ell}} & =-\square \psi_{\mu_{1} \cdots \mu_{\ell}}+\ell \nabla_{\lambda} \nabla_{\left(\mu_{1}\right.} \psi_{\left.\mu_{2} \cdots \mu_{\ell}\right)}^{\lambda} \\
& =-\left(\square+\frac{\ell(\ell+D-2)}{L^{2}}\right) \psi_{\mu_{1} \cdots \mu_{\ell}} .
\end{aligned}
$$

Thus the solutions of 11 must also satisfy the equation 3 for appropriate $M^{(\ell)}$.

We are now in the position to present the solutions of 11 . A priori, $\psi_{\mu_{1} \cdots \mu_{\ell}}^{(\ell)}$ are in general functions of all coordinates, with parameters $E_{0}^{(\ell)}$ and $h_{i}$. However, for non-trivial solutions in $D \geq 4$, we find that

$$
h_{1}=\ell, \quad h_{i}=0, \quad \text { for } \quad i \geq 2,
$$

(In $D=3, h_{1}= \pm \ell$, owing to the fact that $S O(2,2) \sim S L(2, R) \times S L(2, R)$.) The reason for $h_{1}$ being singled out here is due to the specific choice of the definition of positive and simple roots that we adopt, as in [24]. The scalar $\ell=0$ solution is given by

$$
\psi^{(0)}=\Phi \equiv e^{-\mathrm{i} E_{0}^{(0)} \tau}(\cosh \rho)^{-E_{0}^{(0)}},
$$

which is independent of any spherical coordinate. Thus this $\ell=0$ solution is a singlet under the $S O(D-1)$ subgroup of $S O(2, D-1)$. It should be pointed that there exist 
scalar solutions in AdS that form infinite-dimensional representations [25], which we shall not consider in this paper. For general higher spins in $D \geq 4$, we find a recursive relation between the spin- $(\ell+1)$ and the spin- $\ell$ solutions

$$
\begin{aligned}
\psi_{\mu_{1} \cdots \mu_{\ell} \tau}^{(\ell+1)} & =\left.s_{\rho} s_{1} s_{2} \cdots s_{n-1} e^{-\mathrm{i} \phi_{1}}\left(\psi_{\mu_{1} \cdots \mu_{\ell}}^{(\ell)}\right)\right|_{E_{0}^{(\ell)} \rightarrow E_{0}^{(\ell+1)}}, \\
\psi_{\mu_{1} \cdots \mu_{\ell} \rho}^{(\ell+1)} & =\left.\mathrm{i} c_{\rho}^{-1} s_{1} s_{2} \cdots s_{n-1} e^{-\mathrm{i} \phi_{1}}\left(\psi_{\mu_{1} \cdots \mu_{\ell}}^{(\ell)}\right)\right|_{E_{0}^{(\ell)} \rightarrow E_{0}^{(\ell+1)}}, \\
\psi_{\mu_{1} \cdots \mu_{\ell} \theta_{i}}^{(\ell+1)} & =\left.\mathrm{i} s_{\rho} s_{1} s_{2} \cdots s_{n-1} \frac{c_{i}}{s_{i}} e^{-\mathrm{i} \phi_{1}}\left(\psi_{\mu_{1} \cdots \mu_{\ell}}^{(\ell)}\right)\right|_{E_{0}^{(\ell)} \rightarrow E_{0}^{(\ell+1)}}, \\
\psi_{\mu_{1} \cdots \mu_{\ell} \phi_{1}}^{(\ell+1)} & =\left.s_{\rho} s_{1} s_{2} \cdots s_{n-1} e^{-\mathrm{i} \phi_{1}}\left(\psi_{\mu_{1} \cdots \mu_{\ell}}^{(\ell)}\right)\right|_{E_{0}^{(\ell)} \rightarrow E_{0}^{(\ell+1)}}, \\
\psi_{\mu_{1} \cdots \mu_{\ell} \phi_{i}}^{(\ell+1)} & =0, \quad \text { for } \quad i=2,3, \cdots
\end{aligned}
$$

where

$$
s_{\rho}=\sinh \rho, \quad c_{\rho}=\cosh \rho, \quad s_{i}=\sin \theta_{i}, \quad c_{i}=\cos \theta_{i} .
$$

In $D=3$, there is an additional class of solutions with $h_{1}=-\ell$. They are given by the above with $\phi_{1} \rightarrow-\phi_{1}$, and the components with odd number of $\phi_{1}$ index change the sign as well. Starting from the $\ell=0$ solution (16), it is straightforward to generate the solutions for all $\ell$ using the recursion relation (17). The solutions thus generated are necessarily totally symmetric. The transverse and traceless conditions 12 are all satisfied. It is easy to verify that the $\ell=1$ and $\ell=2$ solutions are indeed precisely those obtained in [24] by a brutal-force calculation. The spin-2 modes in $D=3$ and 4 were obtained in [22] and [23] respectively. The spin-3 solution in $D=3$ was also constructed in [26]. (Compare our solutions with the previous known examples in $D=3$, we have $E_{0}=h+\bar{h}$ and $\ell=h-\bar{h}$.) This recursive relation makes it straightforward to write up the explicit solution for any spin- $\ell$ in any dimensions. It is thus unnecessary and also too complex to present the full results for higher $\ell$ and $D$, owing to the proliferation of the components. It is perhaps instructive just to present a particular component, namely

$$
\psi_{\tau \cdots \tau}^{(\ell)}=\left(s_{\rho} s_{1} \cdots s_{n-1}\right)^{\ell} e^{-\mathrm{i}\left(E_{0}^{(\ell)} \tau+\ell \phi_{1}\right)}(\cosh \rho)^{-E_{0}^{(\ell)}} .
$$

The box and the Laplace actions on the solutions are given by

$$
\begin{aligned}
\square \psi_{\mu_{1} \cdots \mu_{\ell}}^{(\ell)} & =\frac{\left(E_{0}^{(\ell)}\right)^{2}-(D-1) E_{0}^{(\ell)}-\ell}{L^{2}} \psi_{\mu_{1} \cdots \mu_{\ell}}^{(\ell)}, \\
\Delta \psi_{\mu_{1} \cdots \mu_{\ell}}^{(\ell)} & =-\frac{\left(E_{0}^{(\ell)}\right)^{2}-(D-1) E_{0}^{(\ell)}+\ell(\ell+D-3)}{L^{2}} \psi_{\mu_{1} \cdots \mu_{\ell}}^{(\ell)} .
\end{aligned}
$$

Compare the first equation above to (3), we have

$$
\left(M^{(\ell)}\right)^{2}=\left(E_{0}^{(\ell)}+\ell-2\right)\left(E_{0}^{(\ell)}-\ell-D+3\right) .
$$


It is perhaps more natural to write $(3)$ as

$$
\left(\square+\frac{\ell}{L^{2}}-\hat{M}^{2}\right) \psi^{(\ell)}=0, \quad \hat{M}^{2}=\frac{\left(E_{0}^{(\ell)}\right)^{2}-(D-1) E_{0}^{(\ell)}}{L^{2}},
$$

where $\hat{M}$ does not depend on $\ell$ manifestly.

Having obtained the solutions corresponding to the highest-weight state of the spin- $\ell$ representation, we can use the negative-root generators to act on the solution to obtain the full multiplet. In $D=4$, we generate $2 \ell+1$ solutions with $h=\ell, \ell-1, \cdots,-\ell+1,-\ell$. The procedure becomes more complicated in higher dimensions. In figure 1, we give an example of how the 16-dimensional spin-3 representation in $D=5$ is generated from the highestweight $(3,0)$ state. Note that $E_{-\vec{\alpha}_{2}}$ and $E_{-\vec{\alpha}_{3}}$ are the two negative-root conjugates of the simple roots associated with the $S O(4)$ subgroup of $S O(2,4)$. The explicit expressions for these generators in our AdS coordinates can be found in [24]. In general, the dimension for the massive spin- $\ell$ representation in $D$ dimensions is given by

$$
n=\frac{(2 \ell+D-3)(\ell+D-4) !}{(D-3) ! \ell !} .
$$
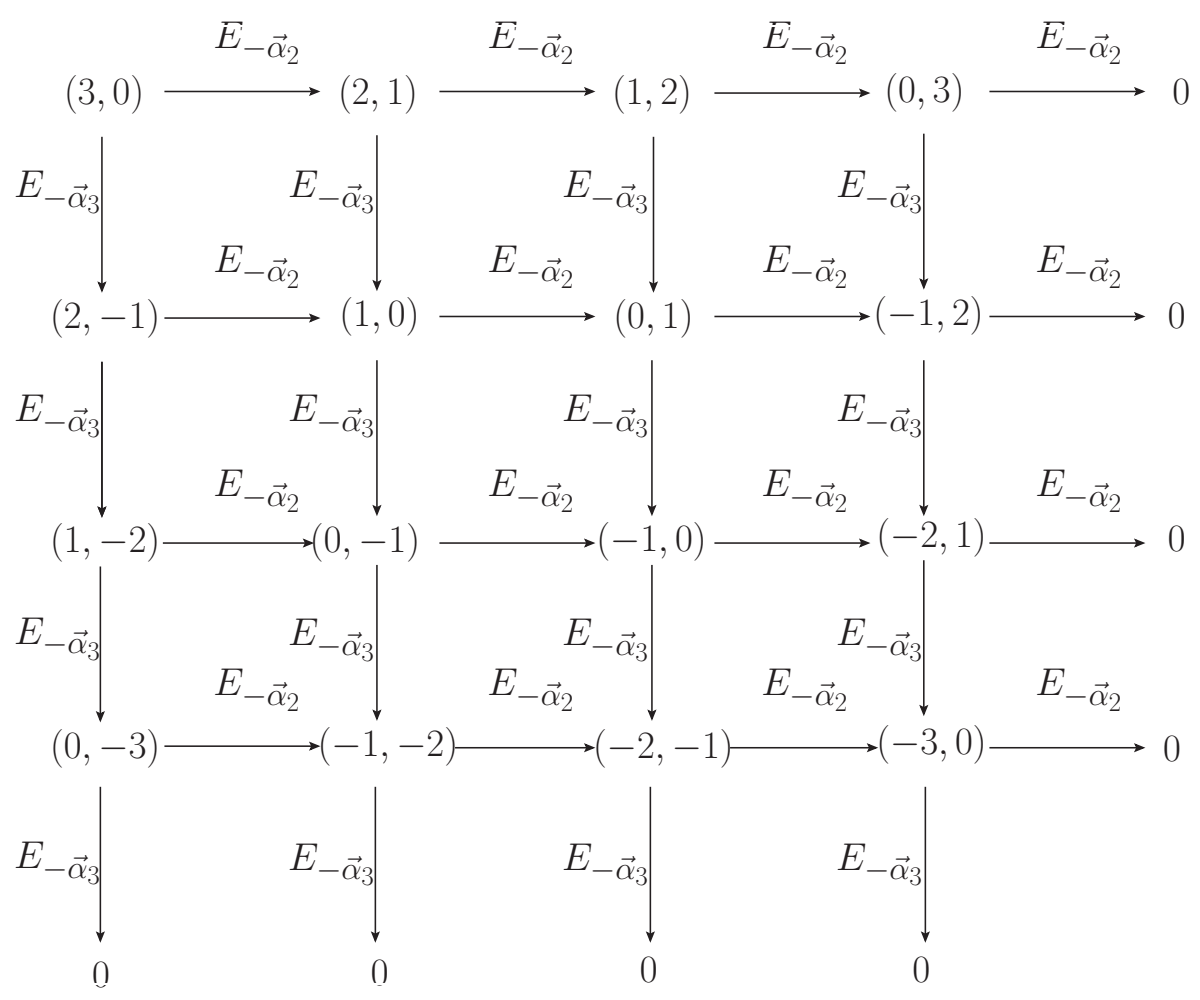

Fig. 1: The generation of the 16-dimensional spin-3 representation of $S O(2,4)$ from the highest (3,0)-state. 
We now study the properties of our solutions. For them to be absent from exponential growth in time, it is necessary that $E_{0}^{(\ell)}$ must be real. It follows from 21 that

$$
\left(M^{(\ell)}\right)^{2} \geq-\frac{(2 \ell+D-5)^{2}}{4 L^{2}} .
$$

This is the generalized Breitenlohner-Freedman (BF) bound for higher-spin fields. The apparent difference of this bound for $\ell=0$ with the usual BF bound is due to the different definitions of the mass. Expressed in terms of $\hat{M}$, defined in 22 , we have

$$
\hat{M}^{2} \geq-\frac{(D-1)^{2}}{4 L^{2}} \text {. }
$$

Although higher spins in AdS backgrounds have the analogous BF bounds, their falloff behavior with negative mass square may differ from that of the scalars. The leading term of the $\psi_{\tau \cdots \tau}(19)$ in the AdS boundary is given by

$$
\psi_{\tau \cdots \tau}^{(\ell)} \sim \frac{1}{r^{E_{0}^{(\ell)}-\ell}},
$$

where $r=\sinh \rho \rightarrow \infty$. The falloff of the AdS black hole is given by $\delta g_{\tau \tau} \sim 1 / r^{D-3}$. In [24], the black hole falloff is used to define the massless higher-spin modes. It is easy to see that indeed the condition

$$
E_{0}^{(\ell)}-\ell=D-3
$$

corresponds precisely $M^{(\ell)}=0$ in 21 . Thus for solutions with $\left(M^{(\ell)}\right)^{2} \geq 0$, it follows from (21) that there must exist a branch that has the falloff faster than that of the black hole.

In the asymptotically-flat space-time, the consistent boundary condition is defined such that no mode should have a falloff slower than the $1 / r^{D-3}$. In solutions that is asymptotic to AdS, more general boundary conditions are allowed, which contain slower falloffs. There is no unique choice for consistent boundary conditions. The strongest and non-trivial boundary condition is that all modes should have the same or faster falloffs than $1 / r^{D-3}$. We call this strong boundary condition.

For solutions that satisfy the bound $(24)$, but with $\left(M^{(\ell)}\right)^{2}<0$, two situations can arise. The first is that $D-5+2 \ell \leq 0$, the solutions satisfy the strong boundary condition. This include the scalar modes in $D=4$ and 5 . For $D-5+2 \ell>0$, the solution has a slower falloff than what is required by the strong boundary condition. It is argued in [27] that the ghost massive graviton in extended gravities with such falloffs could be truncated out by the strong AdS boundary condition.

Finally, we would like to point out that in higher-derivative theories, equations like $\left(\square+\ell / L^{2}-\hat{M}^{2}\right)^{2} \psi_{\mu_{1} \cdots \mu_{\ell}}^{(\ell)}=0$ may arise, in which case there exist log modes which are given by our solutions multiplied by a universal overall factor $i(\tau+\log (\cosh \rho))$. 
To summarize, we consider free higher integer spins in AdS backgrounds in diverse dimensions. We construct explicit solutions corresponding to the highest-weight states of the spin- $\ell$ representations of the $S O(2, D-1)$ isometry groups of the AdS space-times. Such a solution is the eigenstate of the Cartan generators, annihilated by all positive-root generators. The full multiplet can then be obtained by acting on the solution repetitively with negative-root generators. These explicit solutions enable us to obtain the generalized Breitenlohner-Freedman bound for the higher spins, which allow negative mass square. However, we find that the solutions with negative mass square do not in general satisfy the strong AdS boundary condition. Our results should be useful in studying the spectrum and the properties of the linearized modes in AdS gravities coupled to higher spins.

\section{Appendix: Gauge-fixing for massless higher-spin fields}

In this appendix, we examine the gauge fixing of the gauge-invariant equation (4). The procedure does not apply for $\ell=0$. For $\ell=1$, it is given by the standard transverse condition. For $\ell=2$, we adopt the following gauge condition

$$
\nabla^{\mu} \phi_{\mu \nu}^{(2)}=\nabla_{\nu} \phi^{(2)}
$$

where $\phi^{(2)} \equiv g^{\mu \nu} \phi_{\mu \nu}^{(2)}$. Note that in this appendix, we use the notation $\phi_{\mu_{1} \cdots \mu_{k}}^{(\ell)}$ with $k<\ell$ to denote the field for which a trace or multiple traces are taken. It is straightforward to see that applying this gauge and taking the trace of (4), we have $\phi^{(2)} / L^{2}=0$, and we arrive at the transverse and traceless conditions (2). If the theory has no cosmological constant, then this gauge is somewhat singular in that there is no equation for the trace mode $\phi^{(2)}$ at all. This gauge choice was first used in [22] for studying chiral gravity in three dimensions. It was later adopted to study critical phenomena in extended gravities in four [28] and higher dimensions [29].

For $\ell=3$, we consider the following gauge condition

$$
\nabla^{\mu} \phi_{\mu \nu \rho}^{(3)}=2 \nabla_{(\nu} \phi_{\rho)}^{(3)}
$$

which implies that $\nabla^{\mu} \phi_{\mu}^{(3)}=0$. Substituting these into (4) and taking a trace, we find that the transverse and traceless conditions (2) come out naturally.

The situation for $\ell \geq 4$ is different. Let us focus on $\ell=4$. The most general gauge condition is given by

$$
\nabla^{\mu_{1}} \phi_{\mu_{1} \mu_{2} \mu_{3} \mu_{4}}^{(4)}=3 \alpha \nabla_{\left(\mu_{2}\right.} \phi_{\left.\mu_{3} \mu_{4}\right)}^{(4)}+3 \beta g_{\left(\mu_{2} \mu_{3}\right.} \nabla_{\left.\mu_{4}\right)} \phi^{(4)}
$$


where $\alpha$ and $\beta$ are constants to be determined. Substituting this into (4), we find that the derivative terms on $\phi^{(4)}$ cannot be canceled regardless the choices of $\alpha$ and $\beta$, and that the equation for $\phi^{(4)}$, which turns out to be independent of $(\alpha, \beta)$, is given by

$$
\left(\square-\frac{2(D+1)}{L^{2}}\right) \phi^{(4)}=0
$$

It is of interest to note that the scalar trace mode $\phi^{(4)}$ appears massive, compared to (4). It is thus more subtle to eliminate this mode, analogous to the subtlety in eliminating the trace mode in Einstein gravity in the De Donder gauge.

We see that for $\ell=2,3$, the transverse and traceless conditions can be derived from some proper choice of gauging. For $\ell \geq 4$, the situation is more subtle, and we simply impose the conditions (2) by hand.

\section{Acknowledgement}

We are grateful to Haishan Liu, Ergin Sezgin, Zhao-Long Wang for useful discussions. H.L. is supported in part by the NSFC grant 11175269. H.L. is grateful to the Korea Institute for Advanced Study for hospitality during part of the work.

\section{References}

[1] M. Fierz, W. Pauli, On relativistic wave equations for particles of arbitrary spin in an electromagnetic field, Proc. Roy. Soc. Lond. A173, 211-232 (1939).

[2] L.P.S. Singh, C.R. Hagen, Lagrangian formulation for arbitrary spin. 1. The boson case, Phys. Rev. D9, 898-909 (1974).

[3] C. Fronsdal, Massless Fields with Integer Spin, Phys. Rev. D18, 3624 (1978).

[4] C. Aragone and S. Deser, Consistency problems of hypergravity, Phys. Lett. B 86, 161 (1979).

[5] M.A. Vasiliev, Consistent equation for interacting gauge fields of all spins in $(3+1)$ dimensions, Phys. Lett. B243, 378-382 (1990).

[6] M.A. Vasiliev, Progress in higher spin gauge theories, hep-th/0104246.

[7] B. Sundborg, Stringy gravity, interacting tensionless strings and massless higher spins, Nucl. Phys. Proc. Suppl. 102, 113-119 (2001). hep-th/0103247. 
[8] L. Dolan, C. R. Nappi, E. Witten, Conformal operators for partially massless states, JHEP 0110, 016 (2001). hep-th/0109096.

[9] A. M. Polyakov, Gauge fields and space-time, Int. J. Mod. Phys. A17S1, 119-136 (2002). hep-th/0110196.

[10] E. Sezgin, P. Sundell, Massless higher spins and holography, Nucl. Phys. B644, 303-370 (2002). hep-th/0205131].

[11] E. Sezgin, P. Sundell, Analysis of higher spin field equations in four-dimensions, JHEP 0207, 055 (2002). hep-th/0205132.

[12] L. Girardello, M. Porrati, A. Zaffaroni, 3-D interacting CFTs and generalized Higgs phenomenon in higher spin theories on AdS, Phys. Lett. B561, 289-293 (2003). hepth/0212181.

[13] S. Deser, A. Waldron, Arbitrary spin representations in de Sitter from dS/CFT with applications to dS supergravity, Nucl. Phys. B662, 379-392 (2003). [hep-th/0301068].

[14] G. Bonelli, On the covariant quantization of tensionless bosonic strings in AdS spacetime, JHEP 0311, 028 (2003) [arXiv:hep-th/0309222].

[15] M. Bianchi, Higher spins and stringy $A d S_{5} \times S^{5}$, Fortsch. Phys. 53, 665-691 (2005). hep-th/0409304.

[16] D. Sorokin, Introduction to the classical theory of higher spins, AIP Conf. Proc. 767, 172-202 (2005). [hep-th/0405069].

[17] N. Bouatta, G. Compere, A. Sagnotti, An introduction to free higher-spin fields, hepth/0409068.

[18] M.A. Vasiliev, Higher spin gauge theories in any dimension, Comptes Rendus Physique 5, 1101-1109 (2004). hep-th/0409260.

[19] X. Bekaert, S. Cnockaert, C. Iazeolla, M. A. Vasiliev, Nonlinear higher spin theories in various dimensions, hep-th/0503128.

[20] M. Bianchi, V. Didenko, Massive higher spin multiplets and holography, hepth/0502220.

[21] M. Bianchi, P. J. Heslop, F. Riccioni, More on La Grande Bouffe, JHEP 0508, 088 (2005). hep-th/0504156]. 
[22] W. Li, W. Song, A. Strominger, Chiral gravity in three dimensions, JHEP 0804, 082 (2008). arXiv:0801.4566 [hep-th]].

[23] E.A. Bergshoeff, O. Hohm, J. Rosseel, P.K. Townsend, Modes of log gravity, Phys. Rev. D83, 104038 (2011). arXiv:1102.4091 [hep-th]].

[24] Y.X. Chen, H. Lü and K.N. Shao, Linearized modes in extended and critical gravities, arXiv:1108.5184 [hep-th].

[25] V. Balasubramanian, P. Kraus and A.E. Lawrence, Bulk versus boundary dynamics in anti-de Sitter space-time, Phys. Rev. D 59, 046003 (1999) arXiv:hep-th/9805171.

[26] B. Chen, J. Long and J.b. Wu, Spin-3 topological massive gravity, arXiv:1106.5141 [hep-th].

[27] H. Lü, Y. Pang, C.N. Pope, Conformal gravity and extensions of critical gravity, Phys. Rev. D84, 064001 (2011). arXiv:1106.4657 [hep-th]].

[28] H. Lü, C.N. Pope, Critical gravity in four Dimensions, Phys. Rev. Lett. 106, 181302 (2011). arXiv:1101.1971 [hep-th]].

[29] S. Deser, H. Liu, H. Lü, C.N. Pope, T.C. Sisman, B. Tekin, Critical points of Ddimensional extended gravities, Phys. Rev. D83, 061502 (2011). arXiv:1101.4009 [hepth]]. 